\title{
Changes in Hyolaryngeal Movement During Swallowing in the Lateral Decubitus Posture
}

\author{
Byung-Mo Oh, MD, PhD' ${ }^{1}$ Jae Hyun Lee, MD², Han Gil Seo, MD, PhD ${ }^{1}$, Woo Hyung Lee, MD ${ }^{1,3}$, \\ Tai Ryoon Han, MD, $\mathrm{PhD}^{4}$, Seoung Uk Jeong, $\mathrm{MD}^{2}$, Ho Joong Jeong, MD, PhD², Young-Joo Sim, MD, PhD ${ }^{2}$ \\ ${ }^{1}$ Department of Rehabilitation Medicine, Seoul National University Hospital, Seoul National University College of Medicine, \\ Seoul; ${ }^{2}$ Department of Physical Medicine and Rehabilitation, Kosin University College of Medicine, Busan; ${ }^{3}$ Department of \\ Biomedical Engineering, Seoul National University College of Medicine, Seoul; ${ }^{4}$ Department of Rehabilitation Medicine, \\ Gangwon-Do Rehabilitation Hospital, Chuncheon, Korea
}

Objective To investigate the differences in hyolaryngeal kinematics at rest and during swallowing in the upright sitting (UP) and the lateral decubitus (LD) postures in healthy adults, and delineating any potential advantages of swallowing while in the LD posture.

Methods Swallowing was videofluoroscopically evaluated in 20 healthy volunteers in UP and LD postures, based on the movements of hyoid bone, vocal folds, and the bolus head. Parameters included the Penetration-Aspiration Scale (PAS), horizontal and vertical displacement, horizontal and vertical initial position, horizontal and vertical peak position, time to peak position of the hyoid bone and vocal folds, and pharyngeal transit time (PTT).

Results Nine participants were rated PAS 2 in the UP and 1 was rated PAS 2 in the LD ( $=0.003)$ at least 1 out of 3 swallows each posture. The hyoid and vocal folds showed more anterior and superior peak and initial positions in the LD. In addition, swallowing resulted in greater vertical and smaller horizontal displacement of the hyoid in LD posture compared with UP. Time to peak position of the hyoid was shorter in LD. The maximal vertical and horizontal displacement of the vocal folds, and PTT were comparable between postures.

Conclusion The results showed that the peak and initial positions of the hyoid and larynx and the pattern of hyoid movement varied significantly depending on the body postures. This study suggests that the LD posture was one of the safe feeding postures without any increased risk compared with UP posture.

Keywords Deglutition disorders, Posture, Biomechanical phenomena, Hyoid bone, Vocal cords

Received July 12, 2017; Accepted September 15, 2017

Corresponding author: Young-Joo Sim

Department of Rehabilitation Medicine, Kosin University Gospel Hospital, 262 Gamcheon-ro, Seo-gu, Busan 49267, Korea. Tel: +82-51-990-6261, Fax: +82-51-241-2019, E-mail: oggum@hanmail.net

ORCID: Byung-Mo Oh (http://orcid.org/0000-0001-9353-7541); Jae Hyun Lee (http://orcid.org/0000-0002-4927-9722); Han Gil Seo (http://orcid. org/0000-0001-6904-7542); Woo Hyung Lee (http://orcid.org/0000-0003-3413-5950); Tai Ryoon Han (http://orcid.org/0000-0002-7532-9068); Seoung Uk Jeong (http://orcid.org/0000-0002-1267-5879); Ho Joong Jeong (http://orcid.org/0000-0002-0607-2799); Young-Joo Sim (http://orcid.org/00000002-0640-8766).

(c) This is an open-access article distributed under the terms of the Creative Commons Attribution Non-Commercial License (http://creativecommons.org/ licenses/by-nc/4.0) which permits unrestricted noncommercial use, distribution, and reproduction in any medium, provided the original work is properly cited. Copyright $\odot 2018$ by Korean Academy of Rehabilitation Medicine 


\section{INTRODUCTION}

Safe swallowing requires a well-coordinated contraction and relaxation of oropharyngeal muscles [1], which is compromised by diseases affecting the muscles [2] and nervous system [3], as well as by aging [4,5]. Population aging is drawing increased attention to swallowing difficulties, especially in specific elderly populations, such as those in nursing homes [5,6]. Unfortunately, many patients with debilitating diseases [7] and elderly people with dementia [8] find it difficult or impossible to maintain a symmetric, upright sitting (UP) posture for the duration of a meal, resulting in a reclining, supine, or lateral decubitus (LD) posture. Furthermore, people with severe physical disabilities, such as cervical spinal cord injuries, must change postures every 2 hours to prevent pressure ulcers [9]. In this regard, patients may eat meals in the LD.

Studies on the effect of body postures on the swallowing process, primarily focus on the supine posture, using manometry [10], electromyography (EMG) [11], ultrasonography [12] and videofluoroscopic study (VFS) [13,14]. Manometric assessment reveals that different directions of gravitational pull alter the oropharyngeal pressure patterns during swallowing. Compared with the UP, the supine posture yielded greater tongue driving force and weaker hypopharyngeal suction [10]. Although the activation sequence of the laryngeal and suprahyoid muscles remained unaffected by different body postures [11], EMG revealed increased tongue muscle activity in the supine posture [15], which may be a compensatory mechanism to overcome kinetic changes. A supine posture also resulted in major changes in the temporal variables of swallowing, such as pharyngeal delay and transit time for liquids, but not for pudding [14]. Therefore, swallowing in the supine posture may require adaptation to altered gravitational environment, without changing the essential pattern of muscle activation produced by the central pattern generator. In the LD posture, the effect of gravity on the body and food bolus varies from that of the supine posture. Thus, human adaptation may also differ [12-14]. The kinematic differences between supine and LD postures, however, have yet to be extensively investigated.

This study aimed at analyzing differences in hyolaryngeal kinematics at rest and during swallowing in the UP and LD postures in healthy adults, and delineating any potential advantages or disadvantages of swallowing while in the LD posture.

\section{MATERIALS AND METHODS}

\section{Participants}

Twenty healthy Asian subjects (9 men and 11 women) with a mean age of 21.3 years (range, 20-23 years) were recruited for this study. Exclusion criteria were previous history or current symptoms of swallowing difficulty, gastrointestinal disease, or neuromuscular disease. The Institutional Review Board of the Kosin University Gospel Hospital approved this study protocol (IRB No. 11-44). Informed consent was obtained from all participants.

\section{Videofluoroscopic study with different postures}

A thin liquid of diluted barium sulfate (Solotop Suspension 140, Taejoon Inc., Seoul, Korea) with a final concentration of $14 \mathrm{~g} / \mathrm{dL}$ was administered to the participants, in 2-mL doses using a spoon or syringe, in UP and right LD postures, respectively. To minimize radiation exposure, only one consistency was used. Participants sat on a chair with a backrest and assumed a comfortable upright head posture by gazing into an indicator at chest level on the wall in front. While in the LD posture, the head rested on a pillow to maintain a comfortable head and neck posture. Participants held the bolus after the barium delivery and swallowed on receiving a verbal command. Three UP-LD cycles were repeated sequentially (UP-LD-UP-LDUP-LD), totaling 6 swallows per person. An exact lateral view instead of a slight oblique projection maximized the overlap of bilateral mandibular shadows. Continuous mode lateral fluoroscopy (Uromax EX, Shimadzu, Kyoto, Japan) was employed to record video clips at 60 frames per second.

\section{Kinematic analysis}

For subsequent motion analysis, the recorded videos were trimmed using Pinnacle Studio 12.0 (Corel Corp, Ottawa, Canada). Each video clip included frames from 0.5 seconds before the start of the bolus head reaching the lower mandibular margin, to 0.5 seconds after the end of swallowing when the bolus tail completed its passage through the upper esophageal sphincter. A coin measuring $24 \mathrm{~mm}$ in diameter attached to the midline 
under each subject's chin served as a reference ruler.

A researcher digitized the position of the anatomical structures and the food bolus using the Ariel Performance Analysis System (Ariel Dynamics Inc., Trabuco Canyon, CA, USA) frame by frame. Ten video clips of videofluoroscopic swallowing analyses obtained from 10 different participants and copies with slightly modified overall brightness were evaluated for inter-rater and intra-rater reliability. To assess reliability, two researchers with 3 years of experience, who were blinded to information on postures, analyzed these video clips. The anterior-inferior margin of the hyoid bone, the head of the food bolus, and the anterior edge of the subglottic air column were marked. The superior margin of the subglottic air column was selected as a proxy for the vocal folds. The coordinate axes are shown in Fig. 1. All values were calculated using in-house MATLAB script and Statistics Toolbox (R2014b; MathWorks, Natick, MA, USA). We analyzed all the 6 video clips for each subject ( 3 swallows per posture in 2 postures) and averaged all the kinematic parameters per posture for statistical analysis. As a

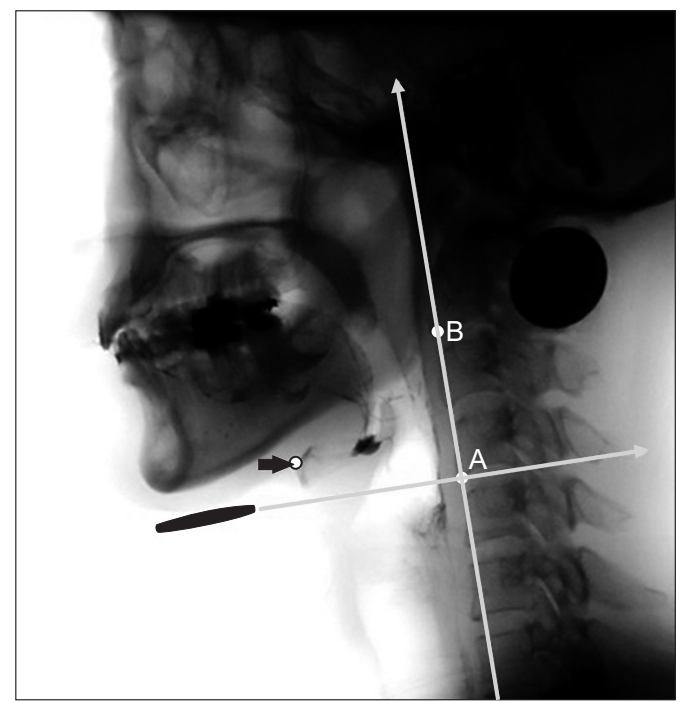

Fig. 1. The coordinate axes of kinematic analysis. The zero point (A) is defined as the anterior-inferior margin of the fourth cervical vertebral body. The vertical axis is defined as the straight line connecting the zero point with the anterior-inferior margin of the second cervical vertebral body (B). The horizontal axis is the axis perpendicular to the vertical axis at the zero point. The movement of the hyoid bone (arrow) is measured with these coordinate axes. result, a dataset consisting of 20 pairs was used for each statistical analysis. The coefficient of variation was calculated for each 3-cycle set of every kinematic parameter to evaluate measurement reliability.

\section{Measurement parameters}

The Penetration-Aspiration Scale (PAS), which is an 8-point observational scale, was used to determine the depth of penetration into the airway [16]. The location of each structure when the bolus head reaches the lower mandibular margin was defined as the initial position. The peak position refers to each structure at the highest (the peak vertical) and the anterior most (the peak horizontal) position. The calculated kinematic parameters included the horizontal and vertical initial positions (HIP, VIP), horizontal and vertical peak positions, maximal horizontal and vertical displacement of the hyoid bone and the vocal folds (Fig. 2A). The maximal horizontal displacement was calculated from the posteriormost to the anteriormost point of the structure during swallowing plus or minus 0.5 seconds. The maximal vertical displacement was defined as the distance between the lowest and the highest positions during swallowing plus or minus 0.5 seconds (Fig. $2 \mathrm{~A}$ ). We measured the location of the bolus head at a time point half-way between the initial and peak vertical positions of the vocal folds. The approach was adopted because, at the peak vertical position of the vocal folds, the bolus head was already in the esophagus in many cases, which was outside of the recorded video clips (Fig. 2B). Velocity was calculated for each set of adjacent frames. Time to peak position was calculated from the point when the bolus head reached the lower margin of the mandible to when the hyoid bone or vocal folds reached the highest position in the vertical or the anteriormost position in the horizontal direction. The pharyngeal transit time (PTT) is the time determined starting from the head of the bolus reaching the lower margin of the mandibular bone to the point when the bolus tail passed through the lower end of the upper esophageal sphincter.

\section{Average trajectories}

To generate an average trajectory of the hyoid bone and vocal folds, subject trajectories were standardized by the temporal normalization employed in our previous study and then pooled [17]. Briefly, each subject's swallowing 
(A)

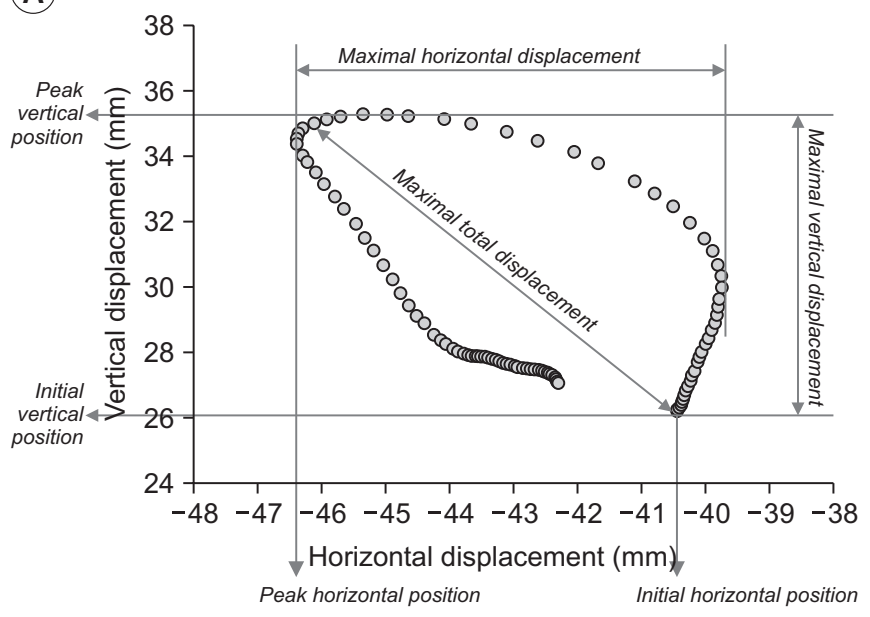

(B)

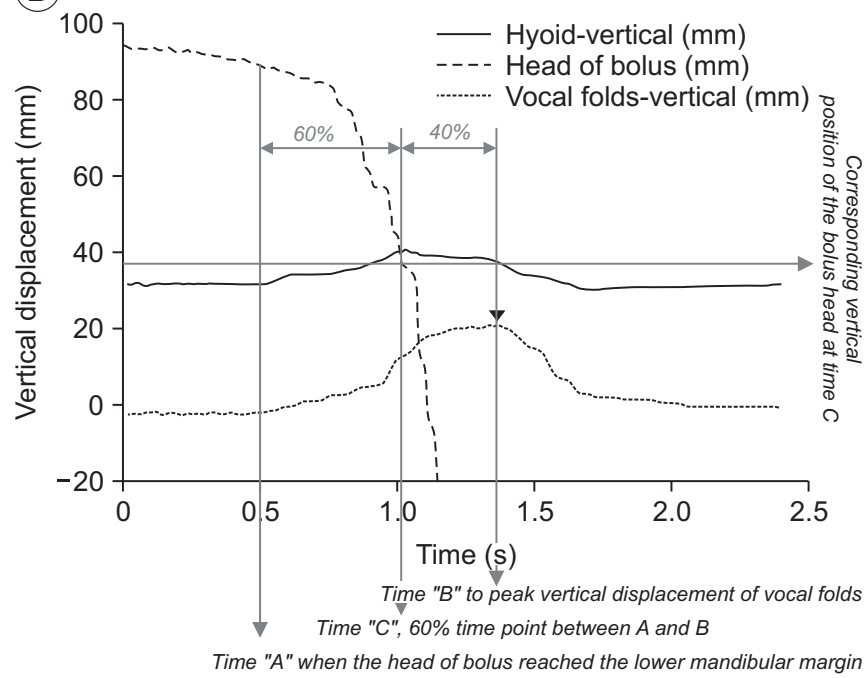

Fig. 2. (A) An exemplary hyoid bone trajectory and the kinematic parameters. Maximal displacement is defined by the difference between the minimal and maximal positions of each structure during swallowing. The maximal 2D displacement is defined as the farthest displacement from the resting position. The 2D displacement may be smaller than the maximal horizontal or vertical displacement because the resting position may not be the lowest or the posteriormost location. (B) The vertical position of the hyoid, vocal folds, and bolus head over time in a representative swallow. The bolus head is already outside of the recorded video coverage when the vocal folds reach the peak vertical position (arrowhead). Instead, the time point $60 \%$ of the way between the time points corresponding to the initial and peak vertical positions is used.

Table 1. Locations of the hyoid bone, vocal folds, and the head of bolus in the upright sitting and lateral decubitus postures

Upright sitting Lateral decubitus $\quad \begin{gathered}\text { Effect size } \\ \text { (Cohen's d) }\end{gathered} \quad$ p-value

\begin{tabular}{|clrrrr}
\hline Hyoid bone & & & & & \\
\hline Initial position & Vertical & $21.37 \pm 8.62$ & $26.20 \pm 9.30$ & 0.54 & $<0.001$ \\
\hline & Horizontal & $-36.16 \pm 4.14$ & $-40.46 \pm 6.29$ & 0.81 & 0.011 \\
\hline Peak position & Vertical & $31.18 \pm 7.02$ & $37.03 \pm 6.57$ & 0.86 & 0.003 \\
& Horizontal & $-44.55 \pm 3.67$ & $-47.74 \pm 6.13$ & 0.63 & 0.002 \\
\hline Vocal folds & & & & & \\
\hline Initial position & Vertical & $-11.75 \pm 8.29$ & $-5.85 \pm 9.51$ & 0.66 & 0.005 \\
\hline & Horizontal & $-29.43 \pm 3.98$ & $-31.90 \pm 5.37$ & 0.53 & 0.001 \\
\hline Peak position & Vertical & $10.20 \pm 6.24$ & $14.61 \pm 5.81$ & 0.73 & 0.004 \\
\hline & Horizontal & $-33.03 \pm 2.57$ & $-35.62 \pm 4.22$ & 0.74 & $<0.001$ \\
\hline Head of bolus & & & & & $<$ \\
\hline $60 \%$ peak position & Vertical & $8.09 \pm 14.1$ & $29.69 \pm 12.78$ & 1.61 & $<0.001$ \\
\hline
\end{tabular}

Values are presented as mean \pm standard deviation.

Negative values denote anterior (horizontal) or inferior (vertical) direction.

Initial position, the position of each structure when the bolus head reaches the lower mandibular margin; Peak position, the position of each structure when it reaches its highest (vertical) or most anterior (horizontal) displacement; $60 \%$ peak position, the location of bolus head at $60 \%$ of the time between the initial and peak vertical position of the vocal folds. 
cycle was normalized temporally by interpolating for 100 time steps. The pooled average trajectory and data dispersion were presented at each time point as a series of mean values and band ranges obtained from the elliptical distributions within $95 \%$ confidence intervals (mean \pm 2 SEM) in both the $\mathrm{x}$ - and $\mathrm{y}$-axes at each time step. As normalization was used only for figure generation, nonnormalized raw data were used for all statistical analysis.

\section{Statistical analysis}

A Wilcoxon signed-rank test was used to compare the maximal displacement, maximal velocities, and PTT between the UP and LD postures, because of the small sample size and non-normal distribution of data. Inter-rater and intra-rater reliability was assessed using an intraclass correlation coefficient (ICC) for each pair of measurements from two copies of the same video clip. The coef- ficient of variation was calculated for each 3-cycle set of every parameter. To compare PAS distribution between postures, $\chi^{2}$ test was used. Differences were considered significant if the $\mathrm{p}$-value was less than 0.05 . Analysis of all data was conducted using SPSS version 14.0 (SPSS Inc., Chicago, IL, USA). Cohen's d was calculated using R [18] and the package "effsize" [19].

\section{RESULTS}

The intra-rater reliability for the maximal displacement of the hyoid bone (vertical/horizontal) and subglottic air column (vertical/horizontal) was $0.997 / 0.916$ and $0.917 / 0.640$, respectively, and the inter-rater reliability was $0.995 / 0.890$ and $0.985 / 0.630$, respectively. The interrater and intra-rater reliability was 0.903 and 0.941 for PTT, respectively. Coefficients of variance for all param-

Table 2. PAS, temporal measurements, and kinematic variables of the hyoid bone and the vocal folds according to body posture during swallowing

\begin{tabular}{|c|c|c|c|c|c|}
\hline & & Upright sitting & Lateral decubitus & $\begin{array}{c}\text { Effect size } \\
\text { (Cohen's d) }\end{array}$ & p-value \\
\hline PAS & & & & & 0.003 \\
\hline 2 & & 9 & 1 & - & \\
\hline 1 & & 11 & 19 & - & \\
\hline Pharyngeal transit time (s) & & $1.36 \pm 0.17$ & $1.32 \pm 0.22$ & 0.20 & 0.191 \\
\hline \multicolumn{6}{|l|}{ Displacement (mm) } \\
\hline \multirow[t]{3}{*}{ Hyoid bone } & Vertical & $11.56 \pm 3.44$ & $12.78 \pm 4.10$ & 0.32 & 0.002 \\
\hline & Horizontal & $10.26 \pm 2.39$ & $9.38 \pm 2.68$ & 0.35 & 0.008 \\
\hline & Total & $8.52 \pm 2.40$ & $7.40 \pm 2.06$ & 0.50 & 0.137 \\
\hline \multirow[t]{2}{*}{ Vocal folds } & Vertical & $23.33 \pm 3.64$ & $22.46 \pm 5.23$ & 0.19 & 0.624 \\
\hline & Horizontal & $5.20 \pm 1.98$ & $5.35 \pm 2.00$ & 0.08 & 0.134 \\
\hline \multicolumn{6}{|l|}{ Maximal velocity $(\mathrm{mm} / \mathrm{s})$} \\
\hline \multirow[t]{2}{*}{ Hyoid bone } & Vertical & $66.94 \pm 24.53$ & $73.65 \pm 31.74$ & 0.24 & 0.218 \\
\hline & Horizontal & $53.28 \pm 20.46$ & $44.49 \pm 18.70$ & 0.45 & 0.108 \\
\hline \multirow[t]{2}{*}{ Vocal folds } & Vertical & $171.01 \pm 40.04$ & $140.95 \pm 48.74$ & 0.67 & 0.006 \\
\hline & Horizontal & $35.06 \pm 9.27$ & $35.24 \pm 10.40$ & 0.02 & 0.911 \\
\hline \multirow[t]{2}{*}{ Head of the bolus } & Vertical & $32.52 \pm 31.56$ & $30.46 \pm 34.78$ & 0.06 & 0.970 \\
\hline & Horizontal & $272.27 \pm 71.45$ & $274.12 \pm 78.32$ & 0.02 & 0.881 \\
\hline \multicolumn{6}{|l|}{ Time to peak position (s) } \\
\hline \multirow[t]{2}{*}{ Hyoid bone } & Vertical & $0.58 \pm 0.22$ & $0.47 \pm 0.16$ & 0.57 & 0.026 \\
\hline & Horizontal & $0.70 \pm 0.16$ & $0.67 \pm 0.13$ & 0.21 & 0.514 \\
\hline \multirow[t]{2}{*}{ Vocal folds } & Vertical & $0.68 \pm 0.13$ & $0.63 \pm 0.12$ & 0.40 & 0.070 \\
\hline & Horizontal & $0.80 \pm 0.46$ & $0.86 \pm 0.27$ & 0.16 & 0.837 \\
\hline
\end{tabular}

Values are presented as mean \pm standard deviation.

PAS, Penetration-Aspiration Scale. 
eters from 3 cycles were comparable between UP and LD postures.

Among the 20 participants, 9 were rated PAS 2 for at least 1 out of 3 swallows in the UP posture and 1 was rated PAS 2 in the LD posture ( $\mathrm{p}=0.003)$. Only one participant exhibited penetration (PAS 2) in both postures. Another participant exhibited PAS 2 penetration in 2 out of 3 swallows in UP posture, and another 8 exhibited PAS 2 penetration in only one of 3 swallows in UP posture. No swallows exhibited penetration/aspiration severer than PAS 2.

Tables 1 and 2 summarize the initial and peak positions, displacement, velocity, and times to peak position of hyoid, vocal folds, and bolus head in the vertical and horizontal directions. There were significant differences in HIP and VIP between the two postures. Displacement of hyoid bone varied significantly (vertical, $p=0.002$; horizontal, $\mathrm{p}=0.008$ ), though the maximal velocity was comparable. The HIP, VIP, and TPP of the hyoid bone and vocal folds in the vertical direction varied significantly between the two postures. Figs. 3 and 4 show the trajectories of the hyoid bone and vocal fold movement during swallowing in the two postures.

\section{DISCUSSION}

The present study evaluated kinematic changes during swallowing in the LD posture, which minimize the effect of gravity on a food bolus as well as the swallowing-

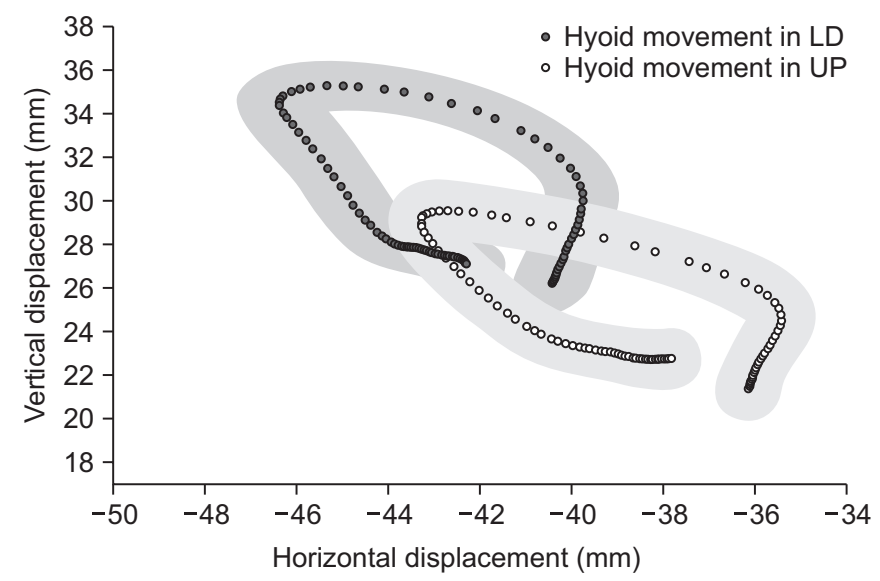

Fig. 3. Average trajectories of the hyoid bone during upright sitting posture (UP) and lateral decubitus posture (LD). In the Cartesian plane at the right upper corner, the asterisk $\left.{ }^{*}\right)$ shows the anterior end of the hyoid bone. related structures. We report that the LD posture moves the resting as well as peak position of the hyoid bone and larynx forward and upward during swallowing. In addition, the trajectory of hyoid movement changes with $\mathrm{LD}$ posture, showing greater superior and smaller anterior displacement during swallowing. In the LD posture, the hyoid reached the peak superior position earlier compared with the UP posture, while PTT was comparable. The LD posture exhibited less penetration than the UP posture.

\section{Resting position of the hyoid bone and larynx}

The resting positions of hyoid bone and larynx were higher and more forward in the LD posture when compared with the UP posture in the present study. However, the resting position of the hyoid bone moves more posteriorly and inferiorly in the supine posture than in the UP posture [13]. This finding suggests a fundamental difference in the effect of supine and LD postures on swallowing kinematics. First, supine and LD postures impose different directions of gravitational force on the hyoid bone, larynx, and food bolus. Supine posture leads to a posterior gravitational pull on the tongue and food bolus, which can ease tongue base retraction and strengthen oral propulsive pumping [10]. Right LD posture, however, does not exert backward or downward gravitational pull, leaving right-directed force on the coronal plane, resulting in minimal effects on the movement in lateral fluo-

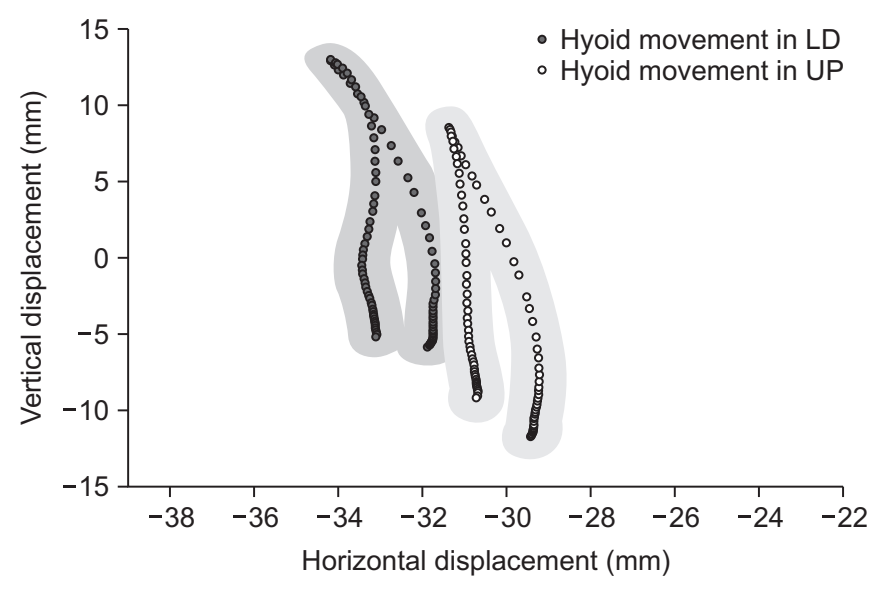

Fig. 4. Average trajectories of the vocal folds during swallowing in the upright sitting posture (UP) and lateral decubitus posture (LD). In the Cartesian plane in the right upper corner, the arrowhead shows the anterior superior point of the subglottic airway column. 
roscopic projection. Thus, LD posture results in minimal gravitational effect on swallowing-related structures, unlike the supine posture. Second, the downward pull of the hyolaryngeal complex by the lung and trachea differs between the supine and LD postures. Since the larynx is connected to the lung via the trachea, and to the hyoid bone by ligamentous structures and muscles [20], the compliance and flexibility of the lung, trachea, and adjacent soft tissues affects hyolaryngeal elevation during swallowing. Adhesion and fibrosis of surrounding soft tissues induce fatigue during swallowing in many head and neck cancer survivors [21]. Additional studies are required to investigate the thoraco-abdominal biomechanics in the supine and LD postures. Third, pre-swallow jaw motion or tongue muscle activity affects the resting position of the hyoid bone and larynx [18]. Compared with the UP posture, the LD posture increases the difficulty in holding the food bolus still, which increases the effort of the tongue just before swallowing. Further research is required to compare the resting hyoid position with and without food bolus in the two body postures.

\section{Maximal displacement of the hyoid and larynx}

Most studies investigating the kinematic effect of the swallowing posture relate to the supine posture $[12,13,15,22]$. However, the effect of supine posture on hyoid bone displacement during swallowing is controversial. While ultrasonography showed no significant difference in hyoid maximal displacement [12], another study reported that the resting hyoid position moves to a more posterior position in the supine posture resulting in a greater anterior displacement during swallowing [13]. The present study reaffirms that $2 \mathrm{D}$ displacement of the hyoid bone remains unchanged in the LD posture. Furthermore, we report a novel finding that the hyoid bone trajectory during swallowing has a different shape, greater upward and smaller forward displacement in the LD posture compared with the UP posture. Ultrasonography might be less precise in evaluating vertical and horizontal hyoid displacement separately due to difficulties in controlling head motion, probe angle, and pressure on the contact surface. Greater vertical displacement in the LD posture can be explained by changing the direction of the gravitational force and the reduction of downward force on the hyoid bone and larynx or strenuous tongue activity in order to compensate for less effective bolus transport.

\section{Time to peak position and velocity}

Another novel finding is that the hyoid reached the peak superior position earlier in the LD posture compared with the UP posture. The PTT was comparable, which is consistent with previous reports $[11,22]$. It may be an epiphenomenon related to increased tongue activity in oral propulsion to accommodate the different direction of gravitational pull. However, considering that temporal patterns of the swallowing-related muscle electromyographic activities are comparable between upright and supine postures [11], a shorter time to peak position is probably related to mechanical changes due to decreased downward force than changes in neuromuscular control. In other words, the LD posture facilitates early peak position of the hyoid bone during swallowing by diminishing the opposing downward force, which is a combination of gravitational pull and traction from the soft tissue, lung, and trachea. Although laryngeal vertical peak velocity was reduced in the $\mathrm{LD}$ posture, the mean vertical velocity until the peak position was reached remained comparable between the UP and LD postures $(p=0.903)$. The lower peak vertical velocity, with no difference in mean velocity of the larynx, suggests an upward acceleration of the larynx in the early phase of swallowing, which might also be related to decreased opposing downward force in an $\mathrm{LD}$ posture.

\section{Clinical implications}

In the present study, the LD posture yielded less frequent penetration (PAS 2) compared with the UP posture (Table 2). It is essential not to overestimate this result because PAS 2 is considered normal in that up to $20 \%$ of apparently healthy young adults reportedly showed PAS 2 penetration [23]. This observation is in line with the aforementioned findings, such as higher location of the food bolus during laryngeal ascension, higher resting and peak position of the hyoid and larynx, and shorter time for the hyoid to reach its peak vertical position. The hyoid showed smaller anterior and greater superior displacement during swallowing in the LD posture. In addition, the resting position of the hyoid and larynx moved farther upward and forward. Molfenter and Steele [24] suggested that a higher peak vertical position of the hyoid bone is related to a decreased risk of penetration. 
Therefore, enhanced vertical displacement and elevated resting and peak hyoid position might facilitate airway protection. Although the difference was small, expedited excursion of the hyoid bone to its peak vertical position may not endanger safe swallowing. Therefore, we suggest that consuming small amounts of liquid in the LD posture does not increase the risk in terms of hyolaryngeal positioning and movement. However, previous studies reported that the LD posture compromises respiratory function in certain patient populations $[25,26]$. Therefore, further studies are required to determine if LD posture was safe for consumption of larger volume of liquid as well as other diet with different textures. In addition, the oral and esophageal phases of swallowing require evaluation to obtain a complete safety profile.

Understanding the changes in the magnitude and direction of the motion of the hyoid bone and larynx with different body postures provides a foundation for personalized treatment in patients who are unable to maintain upright posture. The horizontal travel distance of the hyoid was smaller, but the peak position moved farther forward due to the altered resting hyoid position in the LD than in the UP posture. These results suggest that different body postures change the environment for swallowing exercises, linked to different treatment effects. Thus, planning for anti-gravity exercises in dysphagia management requires consideration of posture effects on hyolaryngeal movement.

\section{Study limitations}

First, the pre-swallow tongue activity might differ between the two postures, particularly because different food delivery methods (spoon vs. syringe) were used. Most participants, however, stayed still while waiting to swallow, which stabilized the hyoid bone position for the initial half second before swallowing (Fig. 2B). In future investigations, a consistent delivery method such as straw drinking will be a more viable option in order to minimize this concern. Second, the findings in young and healthy volunteers require caution until there is clearer evidence of the benefits in older population. Third, the head-to-head comparison between the UP and LD postures while maximizing the statistical power, excluded the supine posture from the study design and may have limited the richness and variety of clinical inferences. Finally, a potential carry-over effect occurred because of the unbalanced design, with which all participants started with UP and ended with LD. A balanced, random sequence design will be more appropriate for the comparison.

\section{Conclusion}

This study is a quantitative analysis of the movement of the hyoid bone and the vocal folds in both the UP and LD postures. The peak and the initial positions of the hyoid and larynx, and the pattern of hyoid movement significantly differed depending on the body postures. Upward movement of the initial and peak hyoid positions and shorter time to peak vertical position suggest that the LD posture may not increase the risk compared with the UP posture, and might be considered one of the safe feeding postures at least for a small amount of liquid. Additional studies are necessary to determine the effect of LD posture in patients with swallowing difficulties.

\section{CONFLICT OF INTEREST}

No potential conflict of interest relevant to this article was reported.

\section{ACKNOWLEDGMENTS}

This study was supported by a grant from the Kosin University College of Medicine (2015).

\section{REFERENCES}

1. Ertekin C, Aydogdu I. Neurophysiology of swallowing. Clin Neurophysiol 2003;114:2226-44.

2. Oh TH, Brumfield KA, Hoskin TL, Stolp KA, Murray JA, Bassford JR. Dysphagia in inflammatory myopathy: clinical characteristics, treatment strategies, and outcome in 62 patients. Mayo Clin Proc 2007;82:441-7.

3. Seo HG, Oh BM, Han TR. Longitudinal changes of the swallowing process in subacute stroke patients with aspiration. Dysphagia 2011;26:41-8.

4. Park YH, Han HR, Oh BM, Lee J, Park JA, Yu SJ, et al. Prevalence and associated factors of dysphagia in nursing home residents. Geriatr Nurs 2013;34:212-7.

5. Achem SR, Devault KR. Dysphagia in aging. J Clin Gastroenterol 2005;39:357-71.

6. Kendall KA, Leonard RJ. Hyoid movement during 
swallowing in older patients with dysphagia. Arch Otolaryngol Head Neck Surg 2001;127:1224-9.

7. Tamura F, Shishikura J, Mukai Y, Kaneko Y. Arterial oxygen saturation in severely disabled people: effect of oral feeding in the sitting position. Dysphagia 1999;14: 204-11.

8. Steele CM, Greenwood C, Ens I, Robertson C, Seidman-Carlson R. Mealtime difficulties in a home for the aged: not just dysphagia. Dysphagia 1997;12:4351.

9. Consortium for Spinal Cord Medicine Clinical Practice Guidelines. Pressure ulcer prevention and treatment following spinal cord injury: a clinical practice guideline for health-care professionals. J Spinal Cord Med 2001;24 Suppl 1:S40-101.

10. Dejaeger E, Pelemans W, Ponette E, Vantrappen G. Effect of body position on deglutition. Dig Dis Sci 1994;39:762-5.

11. Barkmeier JM, Bielamowicz S, Takeda N, Ludlow CL. Laryngeal activity during upright vs. supine swallowing. J Appl Physiol (1985) 2002;93:740-5.

12. Feng X, Cartwright MS, Walker FO, Bargoil JH, Hu Y, Butler SG. Ultrasonographic evaluation of geniohyoid muscle and hyoid bone during swallowing in young adults. Laryngoscope 2015;125:1886-91.

13. Perry JL, Bae Y, Kuehn DP. Effect of posture on deglutitive biomechanics in healthy individuals. Dysphagia 2012;27:70-80.

14. Su HK, Khorsandi A, Silberzweig J, Kobren AJ, Urken ML, Amin MR, et al. Temporal and physiologic measurements of deglutition in the upright and supine position with videofluoroscopy (VFS) in healthy subjects. Dysphagia 2015;30:438-44.

15. Inagaki D, Miyaoka Y, Ashida I, Yamada Y. Influence of food properties and body position on swallowingrelated muscle activity amplitude. J Oral Rehabil 2009; 36:176-83.
16. Rosenbek JC, Robbins JA, Roecker EB, Coyle JL, Wood JL. A penetration-aspiration scale. Dysphagia 1996;11:93-8.

17. Leigh JH, Oh BM, Seo HG, Lee GJ, Min Y, Kim K, et al. Influence of the chin-down and chin-tuck maneuver on the swallowing kinematics of healthy adults. Dysphagia 2015;30:89-98.

18. Bryant KN, Finnegan E, Berbaum K. VFS interjudge reliability using a free and directed search. Dysphagia 2012;27:53-63.

19. Torchiano M. effsize: Efficient Effect Size Computation [Internet]. [place unknown]: The R Foundation; 2017 [cited 2018 May 1]. Available from: https://cran. r-project.org/web/packages/effsize/index.html.

20. Standring S, Berkovitz BK, Ellis H, Gray H. Gray's anatomy: the anatomical basis of clinical practice. 39th ed. Edinburgh: Churchill Livingstone; 2005.

21. Simental AA, Carrau RL. Assessment of swallowing function in patients with head and neck cancer. Curr Oncol Rep 2004;6:162-5.

22. Johnsson F, Shaw D, Gabb M, Dent J, Cook I. Influence of gravity and body position on normal oropharyngeal swallowing. Am J Physiol 1995;269(5 Pt 1):G653-8.

23. Robbins J, Coyle J, Rosenbek J, Roecker E, Wood J. Differentiation of normal and abnormal airway protection during swallowing using the penetration-aspiration scale. Dysphagia 1999;14:228-32.

24. Molfenter SM, Steele CM. Kinematic and temporal factors associated with penetration-aspiration in swallowing liquids. Dysphagia 2014;29:269-76.

25. Palermo P, Cattadori G, Bussotti M, Apostolo A, Contini M, Agostoni P. Lateral decubitus position generates discomfort and worsens lung function in chronic heart failure. Chest 2005;128:1511-6.

26. Teixeira AB, Mathias LA, Saad Junior R. The influence of posture on spirometric values in grade III obese patients. Rev Bras Anestesiol 2011;61:713-9. 